\title{
Formación ciudadana en jóvenes y su impacto en el proceso democrático del país
}

\section{Resumen}

El objetivo de la investigación fue conocer el nivel de formación política, la formación ciudadana, el conocimiento hacia la participación y la actitud hacia la participación ciudadana existente en los jóvenes salvadoreños. La investigación fue de tipo ex post facto con diseño transversal. Los sujetos del estudio son la población joven de El Salvador, siendo representados con una muestra probabilística de 482 sujetos, distribuidos entre los catorce departamentos del país. Entre los resultados principales de la investigación se tiene que, de acuerdo al nivel escolar de los sujetos, se encuentran diferencias significativas en la participación política, la formación ciudadana y la actitud hacia la participación, y que el disponer de un trabajo influye en la actitud que se tiene hacia los procesos políticos nacionales.

Palabras clave: Formación política, formación ciudadana, actitud hacia la participación, participación política, participación juvenil, política, juventud, El Salvador.

\section{Abstract}

The aim of this research was to determine the level of political education, civic education, awareness towards participation and attitude toward the existing citizen participation in Salvadoran youth. The research design was cross-sectional ex post facto. The subjects of the study were young people of El Salvador, being represented with a probability sample of 482 subjects, distributed among the fourteen departments of the country. The main results of the investigation are, that according to educational level of subjects, there are significant differences in political participation, civic education and attitude towards participation, and also that having a job influences the attitude you have to participate in the national political processes.

Keywords: Political education, citizenship education, Attitude towards participation, political participation, youth participation, policy youth, El Salvador. 


\section{Formación política en jóvenes y su impacto en el proceso democrático del país}

Un dicho popular es que "la gente de hoy va a resolver los problemas del mañana". Las escuelas generalmente proveen la preparación esencial para dicha tarea (Westheimer \& Kane, 2004). Aún sobre esta retórica, un currículo que considere la naturaleza de los problemas sociales y las formas en las que un ciudadano puede enfrentarlos raramente logra atención alguna. En vez de enfocarse en enseñar formas en las que la juventud puede participar a través de las instituciones democráticas para construir una mejor sociedad, los currículos actuales se enfocan en la adquisición de habilidades académicas y vocacionales.

La democracia, contrario a lo que una primera idea nos podría sugerir, no es una cualidad humana, sino que es una cualidad social (López, 2007). Es decir, habrá democracia solo si hay sociedad; por tanto, al igual que un humano debe desarrollar sus cualidades para ser integral, así la sociedad debe desarrollar la democracia hasta su máximo para considerarse en verdad como tal.

En el país se han realizado investigaciones previas abordando temáticas similares, destacando la ejecutada por Córdova, Cruz y Seligson (2007), realizada en colaboración con Lapop, Fundaungo, el ludop, el Americas Barometer y la Universidad de vanderbilt, que aborda la cultura de la democracia en El Salvador a escala general. También cabe mencionar el estudio realizado por Alas, Cabrera y Montoya (2011), en el que se describe la situación actual de la juventud en El Salvador, siendo sus hallazgos una referencia importante en la realización de este.

Actualmente el sistema educativo salvadoreño, desde la educación primaria hasta la media, tiene dentro de la malla curricular de estudios sociales contenidos sobre el funcionamiento del Estado, su organización y sus diferentes clasificaciones, así como los diferentes modelos que existen de este contenidos básicos de economía y similares. Sin embargo, y a pesar de tener una formación académica que le proporciona dichos conocimientos, la población juvenil del país mantiene una participación en el ámbito político que va de baja a nula hasta apática en estos procesos, lo cual se refleja en la vida política del país, donde los jóvenes todavía no han podido deshacerse del estigma de niñez, inexperiencia e irrelevancia que tradicionalmente ha caracterizado a este sector. Lo anterior, dado como resultado de la cultura de adultos que todavía domina la sociedad salvadoreña, de la imagen que los mismos jóvenes proyectan de sí mismos como miembros de esta, y de una educación que transmite conocimientos pero que no proporciona las herramientas necesarias para que el educado, sea capaz por sí mismo, de asumir un papel activo en la democracia de la cual es parte. Este conjunto de factores excluye de forma tácita a la juventud de la democracia del país, relegándola a poder dar su opinión en espacios de pseudoparticipación ciudadana dirigidos exclusivamente a los jóvenes, entre los cuales se encuentran los foros y congresos que se realizan cada cierto tiempo, y cuyo objetivo es dejar que los participantes expresen sus ideas con total libertad, algo que, dentro de un país verdaderamente democrático, puede hacerse recurriendo a los canales apropiados y teniendo un impacto verdadero.

El sistema actual del país históricamente no se ha prestado para una verdadera formación política que permita nuevos actores que transformen a la sociedad. Así, siguiendo esta línea, cabe preguntarse: ¿Cuál es el impacto de la formación ciudadana de los jóvenes en la democratización del país?

De tal forma, se estableció como objetivo general de la investigación el determinar el grado de formación ciudadana de los jóvenes salvadoreños, y el impacto que esta tiene en el proceso democrático del país. Para cumplir esta meta, hubo que diseñarla en los siguientes objetivos específicos:

1. Determinar el nivel de formación ciudadana de los jóvenes salvadoreños.

2. Identificar si existen diferencias significativas en el nivel de formación ciudadana de los jóvenes en cuanto a su sexo.

3. Conocer si el nivel educativo incide en el nivel de formación ciudadana que los jóvenes salvadoreños poseen.

4. Comprobar si existe relación entre formación política y formación ciudadana. 
5. Determinar la relación entre conocimiento respecto a la participación ciudadana y las actitudes que los jóvenes tienen hacia la participación.

De esta forma, se hace necesaria la definición de un marco conceptual sobre el que trabajar dichas variables. Así, se hace necesario definir la democracia como una forma de gobierno en la cual todos los ciudadanos adultos tienen igual oportunidad de decisión en los aspectos que afectan sus vidas (Diamond y Plattner, 2006). Idealmente, esto incluye participación igualitaria en la proposición, desarrollo y concreción del proceso de legislación. También abarca condiciones sociales y culturales que permiten la libre e igualitaria práctica de autodeterminación política.

\section{Juventud y democracia}

Para el European Youth Forum (2010), la gente joven de hoy tiene más y mejores oportunidades para el acceso a la información y para ganar conocimiento de la sociedad. Sin embargo, existe una división cada vez más marcada entre las instituciones democráticas y partidos políticos, por un lado, y la sociedad civil, por el otro. El desencanto con la política, la desconfianza con los partidos políticos y la complejidad de los procesos de decisión están creando un déficit democrático.

Los jóvenes de América Latina y el Caribe sufren las consecuencias de estilos de desarrollo excluyentes (Alas, Cabrera y Montoya, 2010). Los modelos económicos adoptados por nuestros países privilegian la inmediatez de la mano de obra, en detrimento de una formación adecuada para las nuevas generaciones que llegan a un mundo para el que no se las prepara para enfrentar.

Donoso (2010), al respecto de la participación ciudadana, considera que la ciudadanía es la expresión de una construcción colectiva que configura las relaciones entre las personas, pero que, al mismo tiempo, son formadas en ese mismo proceso. Vista de este modo, la práctica ciudadana sería el espacio de confluencia entre el individuo y la comunidad. Asimismo, si se entiende que la ciudadanía es una construcción histórico-cultural, esta se trasforma con el transcurrir del tiempo y responde a estructuras sociales diversas.
Molloy (2002) identificó una serie de posibles razones para la apatía de los jóvenes hacia los procesos democráticos, incluyendo:

- las limitadas oportunidades para participar en el proceso político;

- desconocimiento acerca de cómo participar;

- sensación de que tiene mejores cosas que hacer;

- la percepción de que hay una falta de interés en sus puntos de vista, y

- creer que no tiene sentido la votación debido a que aunque se vote, ningún cambio va a darse.

La vinculación de las personas jóvenes con la dimensión política y ciudadana, necesariamente, involucra preguntarse en torno al lugar desde el cual ellos se sitúan en la sociedad (Donoso, 2010). Esto, en tanto se entiende que la participación político-social y la ciudadanía son expresiones de la implicación de los sujetos en el devenir de la comunidad de la que son parte, del papel que individual y colectivamente se asume en dicho devenir y de los derechos que aquella pertenencia comporta. De la misma forma, Tejeda (2010) menciona que la gobernabilidad democrática se refiere a la capacidad de las sociedades para orientar y organizar sus instituciones públicas y sociales, de modo que ofrezcan a las personas más y mejores oportunidades para llevar el tipo de vida que valoran, incluyéndolas en las decisiones que las afectan.

\section{Condiciones para la formación ciudadana}

La formación ciudadana debe entenderse como la adquisición de conocimientos, el desarrollo de habilidades y la incorporación de valores que permitan al estudiante participar, incidir y mejorar la vida de su grupo, su comunidad y su país. Esta formación implica, además, desarrollar su capacidad para la reflexión y el cuestionamiento.

Bermúdez (2008) agrega que el fin de la ciudadanía es el goce efectivo de los derechos humanos de las personas, más allá de los conceptos de caridad, beneficencia y ayuda para la pobreza en el marco de los modelos económicos de desigualdad. Evolucionar en el concepto de ciudadanía 
social, en el marco de la igualdad social, consiste en la realización de los derechos sociales: derecho a un estándar mínimo de bienestar e ingresos, que comprende desde el derecho a una renta mínima hasta el derecho a compartir la riqueza social. Estos derechos garantizarían el sentido y las capacidades para la participación democrática.

\section{Método}

\section{Participantes}

Para la realización del estudio se tomó en cuenta a representantes de la población joven de El Salvador, la cual, utilizando una clasificación estandarizada, comprende la población joven entre los 18 y los 30 años de edad. De este grupo se entrevistó a hombres y mujeres con grados de escolaridad variados, desde educación parvularia hasta educación superior, incluyendo a aquellos con ingresos propios o mantenidos por sus padres, de los catorce departamentos de la República de El Salvador.

En cuanto a las frecuencias de los datos sociodemográficos (ver tabla 1), se tiene que 234 encuestados ( $48,5 \%$ de la muestra) son del sexo masculino, mientras que $248(51,5 \%)$ corresponden al sexo femenino. Asimismo, respecto a los rangos de edad, se tiene que 177 participantes $(36,7 \%)$ están entre los 17 y 20 años de edad; 193 (40\%) están entre 21 y 25 años, y $112(23,2 \%)$ se ubican en el rango de entre 26 y 30 años cumplidos. Para el nivel educativo, entre los valores más bajos se tiene que tres encuestados $(0,6 \%)$ solo contaban con educación parvularia, mientras que 15 (3.1\%) solo tenían educación primaria, mientras que los valores mayores en esta variable incluyeron a la educación secundaria con 42 encuestados (8,7\%); 153 para educación media $(31,7 \%)$ y superior $(55,8 \%)$.

A estas variables se les agregó si el encuestado trabaja o no. Dentro de esta variable, se tiene que 218 encuestados $(45,2 \%)$ están en el grupo de los que sí trabajan, mientras que $264(54,8 \%)$ manifiestan no contar con un empleo fijo.

\section{Universo y muestra}

El universo del estudio lo conforman los jóvenes salvadoreños de entre 18 y 30 años, quienes, de acuerdo con el Censo
Nacional de Población y Vivienda (2007), suman más de 500 mil habitantes. De tal manera que, para el cálculo de la muestra, se utilizó la fórmula para poblaciones finitas, asignándose un nivel de confianza del $97 \%$ y un margen de error del $5 \%(Z=1.96)$, obteniéndose un valor de 482 , mismo que fue utilizado para un muestreo estratificado entre los catorce departamentos de El Salvador.

\section{Diseño y tipo de estudio}

La investigación fue de tipo ex post facto con diseño transversal (Montero y León, 2007), constituida del diagnóstico de una situación ya existente a partir de una hipótesis dada, sin tener la oportunidad de manipular la variable independiente utilizando un post-test sin grupo control.

\section{Instrumento de recolección de datos}

El instrumento utilizado fue un cuestionario de tipo encuesta construido en escala de Likert con cinco niveles que incluyeron desde 1 = nada, 2 = un poco, 3 = suficiente, 4 = bastante y $5=$ completamente. La prueba estuvo constituida por treinta ítems construidos para medir cuatro factores que incluyeron la formación política, la formación ciudadana, el conocimiento de la participación y la actitud hacia la participación. Sobre estos ítems se hizo un análisis inicial que mostró que todos tienen un coeficiente de correlación de ítem total corregido adecuado, siendo el valor de media más bajo el del ítem 8: Ha participado alguna vez en procesos de elaboración de propuestas de ley $(\mathrm{M}=1.65$; $\mathrm{DT}=1.099)$, y el valor más alto el correspondiente a Guarda respeto hacia las demás personas $(\mathrm{M}=3.93 ; \mathrm{DT}=1.188$ ). Al realizarse la prueba de confiabilidad al instrumento, se obtuvo un valor para alfa de la escala total de 0.922 , con un valor para el factor formación política de .915, mientras que para formación ciudadana se obtuvo .831. Por otro lado, para la escala de conocimiento ciudadano se obtuvo un alfa de .870 y para la de actitud hacia la participación se obtuvo un valor de .843 .

Al realizar el análisis factorial para los cuatro dimensiones y aplicarse la prueba de medida de adecuación muestral de Kaiser-Meyer-Olkin, se obtuvo un valor de 0.919 , que indicó la adecuación de los datos y el grado de correlación entre 
ellas como positivo, complementándose con una prueba de esfericidad de Bartlett $(x 2=6988.20 ; p=0.000)$. El análisis se realizó respecto a los cuatro factores previamente prefijados, utilizando una rotación de tipo Varimax para obtener la solución de los componentes. Del resultado, se obtuvo que los cuatro factores utilizados explican el $58,6 \%$ de la varianza total.

\section{Procedimiento}

Se hizo la recolección de datos en lugares públicos que incluyeron plazas, parques y colonias de las ciudades en las que se aplicaron los cuestionarios. Como discriminación a priori de los sujetos, los encuestadores preguntaron la edad a los participantes antes de proceder con la aplicación de pruebas, procediendo a explicar a los participantes el propósito del cuestionario y dándoles la opción de contestarlo ellos mismos, o que el encuestador les preguntara los ítems en voz alta para rellenar los datos. La mayoría de la población se inclinó por la segunda opción, al manifestar que se tenía poco tiempo para la participación en el proceso. Para el procesamiento de la información, se construyó una base de datos en el programa IBM SPSS Statistics v.19. Los datos fueron tabulados por el equipo de estudiantes miembros del programa de ayudantes de investigación de la Utec.

\section{Resultados}

De acuerdo con los resultados obtenidos del contraste de medias entre los valores de cada una de las variables medidas por los diferentes ítems, se tiene que, para el factor de formación política, tanto mujeres como hombres, manifiestan sentirse medianamente identificados con los ideales de los diferentes partidos políticos. Sin embargo, la tendencia es baja respecto a la participación en actividades políticas en general. De acuerdo con las estadísticas obtenidas, no existe diferencia en cuanto al sexo en ninguno de los cuatro factores explorados, ya que ítems, como siente entusiasmo de votar, muestran comportamientos similares entre hombres ( $M=2.56$; $D T=1.373)$ y mujeres $(M=2.53$;
$\mathrm{DT}=1.301)$, mostrando ambos tendencias de un entusiasmo entre moderado y bajo por el ejercicio del sufragio.

Respecto a la edad, para el factor de formación política, existe una tendencia al aumento del interés en esta conforme es mayor la edad de los individuos. Así, el ítem Se siente identificado con los ideales de un partido político en específico, para el grupo de 17 a 20 años, la población manifiesta poco interés $(\mathrm{M}=1.77$; $\mathrm{DT}=1.040)$; mientras que para la misma pregunta, los grupos de 21 a 25 años $(\mathrm{M}=2.24 ; \mathrm{DT}=1.365)$; y 26 a $30(\mathrm{M}=2.40 ; \mathrm{DT}=1.402)$. muestran sentirse identificados moderadamente con los ideales de su partido político. Comportamiento inverso muestra el ítem de Ha aprendido sobre sus derechos y deberes como ciudadano en la escuela, donde el valor más alto apareció en el grupo de 17 a 20 años ( $M=3.78$; DT = 1.209).

\section{Diferencias entre factores según variable sociodemográfica}

Para establecer diferencias entre grupos, y de concordancia con los resultados de la prueba K-S para una muestra, se procedió a realizar la prueba de hipótesis por medio de pruebas no paramétricas. Así, para contrastar las variables Sexo y Cuenta con un trabajo con los diferentes factores identificados, se utilizó la prueba $U$ de MannWhitney complementada con $\mathrm{W}$ de Wilcoxon, que permiten establecer si existen diferencias significativas entre dos grupos; masculino y femenino para Sexo, sí trabaja y no trabaja para Cuenta con un trabajo. De tal forma, se encontró que el sexo no tiene incidencia en ninguno de los factores estudiados, mientras que para Trabaja, se obtuvo que existen diferencias en cuanto a la formación política ( $p$ $=0.002)$, a la formación ciudadana $(p=0.05)$, y actitud hacia la participación $(p=0.015)$, siendo el factor de conocimiento sobre participación el que no reflejó diferencias significativas $(p=0.551)$. De tal forma, se infiere que la condición de trabajo de los jóvenes salvadoreños incide en su nivel de participación política, formación ciudadana y en una mejor actitud hacia la participación en el proceso democrático de El Salvador (ver tabla 1). 
Tabla 1.

Resultados de prueba $U$ de Mann Whitney en función de sexo y condición laboral

\begin{tabular}{|c|c|c|c|c|}
\hline $\begin{array}{c}\text { Variable } \\
\text { agrupación }\end{array}$ & Factores & U de Mann Whitney & W de Wilcoxon & Sig. \\
\hline \multirow[t]{4}{*}{ Sexo } & Formación política & 27152.500 & 58028.500 & 0.285 \\
\hline & Formación ciudadana & 26258.00 & 57134.00 & 0.098 \\
\hline & Conocimiento de participación & 27618.500 & 54646.500 & 0.448 \\
\hline & Actitud hacia la participación & 27930.00 & 58806.00 & 0.581 \\
\hline \multirow[t]{4}{*}{$\begin{array}{c}\text { Condición } \\
\text { laboral }\end{array}$} & Formación política & 23859.500 & 58312.500 & 0.02 \\
\hline & Formación ciudadana & 25594.500 & 60047.500 & 0.05 \\
\hline & Conocimiento de participación & 27656.500 & 51527.500 & 0.551 \\
\hline & Actitud hacia la participación & 24871.500 & 59324.500 & 0.01 \\
\hline
\end{tabular}

* La diferencia de medias es significativa a $p<0.05$

Para el análisis de las variables de tres o más niveles, que incluyen la edad y la escolaridad, se recurrió a la prueba de Kruskall-Wallis, que permitió comparar los grupos que las conforman. Esta prueba se corroboró por medio de un Anova de factor con una prueba post hoc de tipo Scheffé para establecer entre qué grupos existen las diferencias. Así, para la edad, se obtuvo que la variable edad es significativa respecto a la formación política $\left(X^{2}=14.430 ; p=0.001\right)$, formación ciudadana $\left(X^{2}=26.604 ; p=0.000\right)$ y la actitud hacia la participación $\left(X^{2}=30.068 ; p=0.000\right)$; es decir, el grupo de 17 a 20 años mostró menos interés por la política y la formación ciudadana que los individuos de 21 a 25 años, quienes muestran actitudes y comportamientos similares a las de los individuos en el rango de 26 a 30 años. Los datos de la prueba paramétrica confirman dichos hallazgos (ver tabla 2). 
Tabla 2.

Prueba de significación Kruskall-Wallis y Anova para factor edad

\begin{tabular}{lcccc}
\hline \multirow{2}{*}{ Factor } & \multicolumn{2}{c}{ Kruskal-Wallis } & Anova & Sig. \\
\hline Formación política & $\mathbf{X}^{2}$ & Sig. & F & 0.00 \\
Participación ciudadana & 14.430 & 0.001 & 15.456 & 0.00 \\
$\begin{array}{l}\text { Conocimiento sobre } \\
\text { participación }\end{array}$ & 26.604 & 0.000 & 13.193 & 0.185 \\
Actitud hacia la participación & 3.484 & 0.175 & 1.696 & \\
\end{tabular}

* La diferencia de medias es significativa a $p<0.05$

\section{Discusión}

En una primera aproximación, los resultados de la investigación sugieren que en la población encuestada existe poco interés hacia la participación política, lo cual confirma lo propuesto por Preciado (2009), donde se encontró que entre los jóvenes mexicanos existe una tendencia similar. Por otro lado, al analizar los resultados de las pruebas inferenciales, se tiene que un aspecto fundamental de dicha relación es la existencia de diferencias en cuanto a los aspectos de formación política y ciudadana entre aquellos jóvenes que poseen un empleo y aquellos que no.

Este fenómeno es consistente con los hallazgos del Instituto Nacional Demócrata para Asuntos Internacionales (NDI, 2009), que dice que, en el caso de los nicaragüenses, existe un menor interés en la política y menos confianza, también manifestaron menor interés en participar en sus comunidades y menor grado de actividad en la política; sin embargo, difieren en parte de aquellos presentados por la Encuesta de Juventud de Chile (2010), donde los jóvenes manifiestan tener un papel importante en la consolidación de la democracia de su país.

Los hallazgos también difieren con los datos presentados por Córdova, Cruz y Seligson (2006), que encontraron diferencias entre hombres y mujeres en cuanto a la concepción de democracia, mostrando los hombres una definición normativa de la democracia que se repetía más veces que en las mujeres. En el estudio no se encontraron diferencias significativas entre hombres y mujeres en cuanto a ninguno de los factores analizados. Sin embargo, si apoyan los hallazgos en cuanto a la definición de democracia, de acuerdo con el nivel educativo, mostrando una tendencia a considerarla más compleja mientras mayor nivel educativo se posee.

Por otro lado, la formación política orientada al partidismo imperante en el país incide levemente en la formación de valores ciudadanos, pero no es la que los define como tales; y esta relación genera en los jóvenes una apatía leve hacia los procesos de participación, la cual se evidencia en el factor de actitud hacia la participación.

El factor que ha mostrado menos resultados favorables es el de conocimiento hacia la participación, que solamente ha mostrado ser significativo respecto a la escolaridad. En los resultados se manifiesta que solo los jóvenes con niveles más altos de educación son los que perciben tener algún nivel de capacidad para ejercer la participación ciudadana, mientras el resto, a pesar de contar con los mismos derechos fundamentales, no se consideran capaces de participar, y, de hecho, están incapacitados para ello; lo que también incide en la actitud hacia la participación que la mayoría demuestra. 
De tal forma, el estudio ha arrojado datos de una juventud ávida de participar en el rumbo de su país, pero que no cuenta con las herramientas conceptuales para hacerlo, lo que trunca dichos ánimos. La actitud hacia la participación ciudadana mejorará, en tanto que las condiciones sociales y económicas de los jóvenes sean garantizadas, y que el sistema de partidos políticos se oriente menos a la formación de militantes y más a la formación de ciudadanos salvadoreños que trabajen por mantener el país.

\section{Recomendaciones}

- Es conveniente fortalecer los programas de inclusión social juvenil orientados a la difusión del conocimiento sobre derechos y deberes ciudadanos. Un mayor conocimiento de estos es necesario para el fomento de una actitud positiva hacia la participación, condición necesaria para la generación de una verdadera cultura democrática.

- La Política Nacional de Juventud es un instrumento de los jóvenes salvadoreños para asegurar una inserción social y económica efectiva. Aquella detalla múltiples propuestas para asegurar la posibilidad de los jóvenes de ejercer una participación ciudadana plena. Sin embargo, ante un amplio desconocimiento de los medios, alcances y tipos de participación e incidencia democrática que los jóvenes sufren, estas propuestas no pueden llegar a concretarse para los grandes sectores de la población. De tal forma, se recomienda incluir expandir la formación en derechos y deberes ciudadanos que actualmente contempla el currículum de los diferentes niveles educativos nacionales.

La investigación refleja que existe una tendencia generalizada en la población juvenil a confundir la participación político-partidista con la formación ciudadana. Existe un sector dentro de la población estudiada que ha nacido y crecido dentro de una línea ideológica determinada, hecho que genera determinadas actitudes y preconcepciones hacia la participación ciudadana en general. Es recomendable establecer un sistema de formación/información que regule a las juventudes partidistas y que privilegie la preferencia política por criterio y no por herencia.

\section{Referencias}

Alas, W., Cabrera, S. \& Montoya, A. (2011). “Una mirada a la juventud. Contextos, condiciones y desafíos en Guatemala, El Salvador y Nicaragua". Facultad Latinoamericana de Ciencias Sociales, programa El Salvador Flacso. ISBN-978-99923-33-35-8.

Bermúdez Aspirillia, Maritsela (2008). Formación ciudadana para los jóvenes. Disponible en http://www.educacionenvalores.org/ spip.php?article2277.

Córdova, R., Cruz, J. \& Seligson, M. (2006). Cultura política de la democracia en El Salvador. LAPOP. Disponible en http://www. uca.edu.sv/publica/iudop/libros/cultura2006.pdf

Craddock, A. (2005). Differences in gender and civic education in Ukraine. European Consortium of Political Research, Hungary. Disponible en http://www.civiced.org/pdfs/research/ CraddockBudapest.pdf.

Diamond, L. \& Plattner, M. (2006). Electoral systems and democracy. Farrell. Disponible en http://books.google. com.sv/books/about/Electoral_systems_and_democracy. html?id=4BL5WqHHVrwC\&redir_esc=y .

Donoso, R. (2010). 6ta Encuesta Nacional de Juventud. Ministerio de Desarrollo social de Chile. Disponible en http://www.injuv.gob. cl/injuv2010/6_encuesta.

European Youth Forum (2011). Youth and democracy. Disponible en http://www.coe.int/t/dg4/youth/Partners/European_youth_ forum_en.asp.

Fundación Rafael Preciado (2009). Valores ciudadanos, partidos políticos y desarrollo de la democracia. Disponible en http://www.fundacionpreciado.org.mx/Reflexion/PDF/P_ reflexion66.pdf.

Interamerican Development Bank (2007). Democracies in Development: Politics and reform in Latin America. International Institute for Democracy and Electoral Assistance, Hardvard University.

Ministerio de Educación Español (2009). Los valores y la formación ciudadana. Debate educativo. Disponible en http:// debateeducativo.mec.es/pdf/d0.pdf.

Núcleo de Conocimientos y Formación Básicos que debe proporcionar el Bachillerato de la Unam, NCFB-Unam (2000). Formación Ciudadana. Disponible en http://www.cab.unam. mx/Documentos/NCFBa/formciudadana/formciudadana.pdf.

Padilla, G. (2002). Juventud y cultura política. Flacso, programa El Salvador. ISBN 99923-33-20-0.

Programa de las Naciones Unidas para el Desarrollo (2004). La democracia en América Latina: Hacia una democracia de ciudadanas y ciudadanos. PNUD. ISBN- 950-511-940-2. 
Programa de las Naciones Unidas para el Desarrollo (2010). Informe sobre el Desarrollo humano El Salvador 2010: De la pobreza y el consumismo al bienestar de la gente. ISBN 978-9992355-40-4.

Snyder, R \& Samuels, D. (2006). Devaluing the vote in Latin America. Journal of Democracy, Volumen 12, número 1. Disponible en http://www.colmex.mx/centros/cee/long-run/lecture6/ David\%20Samuel,\%20Devaluating\%20the\%20vote.pdf.

Tejeda, E. (2010). Gobernabilidad y convivencia democrática en América Latina y El Caribe: Jóvenes en el mapa. Flacso \& Aecid. Disponible en http://www.flacso.org/fileadmin/ usuarios/documentos/mas_documentos/Investigaciones\%20 Tem\%C3\%A1ticas/ETejeda.pdf.

Unesco (2008). Convivencia democrática, inclusión y cultura de paz: Lecciones desde la práctica educativa innovadora en
América Latina. Disponible en http://unesdoc.unesco.org/ images/0016/001621/162184s.pdf.

Usi, E. (2011). Formación política para promover la participación política de los pueblos indígenas. Disponible en http://www. dw-world.de/dw/article/0,,15533549,00.html.

Vidal Fernández, F. (2008). El modelo Cohen de formación política. Universidad Pontificia de Comillas. Disponible en http://www. iglesiaviva.org/234/234-12-VIDAL.pdf.

Westheimer, J. \& Kahne, J. (2004). What kind of citizen? The politics of educating for democracy. Civic survey. Disponible en http:// www.civicsurvey.org/what_kind_of_citizen.pdf.

Whitehead, L. (2008). Variabilidad en la aplicación de derechos: Una perspectiva comparada. Democracia y Estado. PNUD, Contribuciones al Debate. ISBN 978-9972-612-30-5. 